\title{
DEVELOPMENT OF A LOCATION-BASED MOBILE APPLICATION FOR SMARTPHONES USING OPEN-SOURCE TECHNOLOGIES
}

\author{
Taner Şahin Babaoğlu ${ }^{1}$, İsmail Tarık Baltacı ${ }^{1}$ and Hüseyin Pehlivan ${ }^{1}$ \\ ${ }^{1}$ Gebze Technical University, Kocaeli, 41400, Turkey \\ hpehlivan@gtu.edu.tr
}

KEY WORDS: Smartphone, Mobile Application, GNSS, Smartphone Positioning, Android, IOS.

\begin{abstract}
:
Smartphones capable of receiving Global Navigation Satellite Systems (GNSS) signals and location-based mobile applications have been finding new usage areas day by day. The most popular mobile applications have become a part of our life in many ways, especially in spatial data collection, logistics, sports activities, routing, navigation, information exchange, shopping, emergencies and entertainment. The location-based feature has been the characteristic of the apps realized in real-time or near real-time. Within the scope of this study, a mobile application that can be used on smartphones with the Android operating system and is designed to save time and location information during the desired time and sampling interval and send it to the desired e-mail address is presented. The development stages of this location-based mobile application developed using open-source codes and its use on smartphones were shown. The usage areas of the application and a realized test study were explained.
\end{abstract}

\section{INTRODUCTION}

\subsection{General Instructions}

Smartphones and mobile applications have become a significant part of our daily life. It strengthens this position with new usage areas such as having instant information, communicating with our friends and family, navigation, shopping and entertainment that are increasing continually. As of today, there are more than 2.7 billion smartphone users in the world. The users also spend most of their time (approximately $90 \%$ of the time) using various applications (Statista, A., 2021). And also, there are more than 2.7 million mobile applications in the Google Play store and more than 2.2 million in the App Store as it is known. However, on average, 9 applications are used daily, the other 30 applications per month (Statista, B., 2021). For these reasons, we can also talk about the numerous mobile applications produced but not used. In order for the developed mobile applications not to be left idle, they must first draw attention and fulfil an important function. For this purpose, the number of mobile applications that attract the attention of smartphone users and gain commercial value is increasing day by day while new solutions are coming for the new problems

The most popular and widely used mobile applications rely on current map data and instant location information. We can list the map and location-based application areas as follows:
1. Hiking
2. Instant monitoring of taxis and ambulances
3. Emergency search and rescue
4. Finding directions in daily touristic trips,
5. Tracking and tracking in logistics
6. Data collection in scientific studies,
7. Data collection in the observation and monitoring of natural life
8. Planning and other studies in forest areas
9. Organization and planning in sports activities
10. Discovery and travel activities

The use of dual-frequency GNSS receivers in mobile phones will be more preferred and used in data collection for scientific purposes, in addition to their current use (Zhang et al., 2018, Warnant et al., 2018, Robustelli et al., 2019). In this sense, the ability of recording data with high accuracy by using the advantages of mobile devices will bring many new usage areas (Amit and Vineet, 2021, Paziewskia et al., 2021). For this purpose, in this study, a mobile application was developed that records the global location and time information for a certain period of time and with the desired sampling interval, and sends the recorded data to the e-mail address. It has aimed to evaluate the potential benefits of GNSS data obtained using such a mobile application.

\section{ADVANCES IN SMARTPHONE POSITIONING}

Providing instant location information in all weather conditions with GNSS has inspired many new applications. Spatial information, especially nature conservation, research on environmental issues, management of forest areas, person/people tracking and orientation, is now needed more than ever (Taylor et al., 2002, Holopainen et al., 2014, Sanglyne, 2015, Bettinger et al., 2019).

Before GNSS, it had not been so easy to create a defined position in a coordinate system. However, after the satellitebased systems, it has become easier and cheaper to produce location data, although it has some limitations (McGaughey et al., 2017; Brach et al., 2019, Merry and Bettinger, 2019). Nowadays, GNSS is the unrivalled, and most popular geolocation method due to its advantages in positioning, although it has some limitations. Real-time or 'near-real-time location information, independent of current geodetic points, has facilitated simple navigation, easier positioning of management points, assessment of property boundaries, 
mapping of areas, and many other studies (Bettinger et al., 2019).

With the adoption of GNSS in mobile devices, more complex solutions and combinations of GNSS use in mobile applications continue to emerge, including geofencing, activity recognition and others (Keefe et al., 2019). There is a tendency to use smartphones due to low-cost receivers and continuity features in collecting spatial data needed for many different purposes.

Smartphones use a feature called "assisted GPS" (A-GPS) when determining location (van Diggelen, 2009). In particular, it uses the ability of smartphones to communicate with the Internet to obtain information about satellite signals that is supposed to be at the approximate position of the smartphone. Thus, the receiver does not have to search for all possible signals and the initial correction time can be shortened (Zandbergen and Barbeau, 2011). Most mid-range and high-end smartphone GNSS receivers can receive signals from multiple satellite systems.

\section{A MOBILE APPLICATION DEVELOPMENT PROCESS}

As of 2021, the commercial value of mobile applications has reached gigantic proportions (Statista, B., 2021, Jobe, 2013). This situation brings with it competition and development. In general, we can list the six main stages of successful mobile application development as follows.

1. Strategy

2. Analysis and Planning

3. UI/UX (User Interface/User Experience) Design

4. Application Development

5. Test Phase

6. Deployment and Support

(these stages can be increased or decreased)

The first stage of the mobile application development process is the determination of the strategy. Within the scope of the determined strategy; The target application's user audience, competitive environment, goals and objectives and an appropriate mobile platform should be determined (Invonto 2021, Yamakami, 2005, Heitkötter et al. 2013).

At the second stage, the application idea is shaped and turned into a real project. Analysis and planning begin with defining use cases and determining detailed functional requirements (Ohrt and Turau, 2012).

In stage 3, a mobile application, UX refers to how the application works, UI refers to how it looks. Well designed UX and UI are very significant. The positive impact and popularity of the application on the user largely depends on this.

Stage 4 covers the planning process and is very important. Before starting an application development, the following should be done:

- The technical architecture should be defined,

- A technology stack must be selected

- Development milestones should be defined.

A typical mobile app project consists of three integral parts: backend/server technology, API(s) and mobile app frontend.

Stage 5 covers the testing process. In order for the mobile application to provide a quality solution, it must pass user experience testing, functional testing, performance testing and security testing methods.
Stage 6 covers the distribution and updating of the prepared and tested application. Publishing a developed mobile application means sending it to app stores. Requires submission to Apple App Store for iOS and Google Play Store for Android. A developer account is necessary for the Apple App Store and Google Play Store. Once mobile apps become available in app stores, they are supposed to be constantly followed by technological developments and routinely updated for new mobile devices and operating system platforms.

\section{DEVELOPING A MOBILE APPLICATION}

A mobile android application named 'Location Every Second' (LES) was developed within the scope of this study. It is aimed to easily record the location data as a function of the determined sampling interval with time and send it to the desired e-mail address for analysis by facilitating data collection online or in near real-time in the app. This application can be accessed and downloaded, with the name 'Location Every Second', from the Google Play Store by anyone without age restrictions. It is aimed to be easy and practical to use with its uncomplicated, simple and understandable interface. This android application uses the geographic coordinates generated by the mobile device it is installed on. The LES application provides data with geographic coordinates optionally once per second or with the selected sampling interval. This information is saved as a file and can be sent to the specified e-mail user if desired.

Our application is created in java programming language on the Android studio platform which was used as a software development kit, and no database is used. Interface operations are designed using both Android Studio and Adobe Photoshop CC 2019.

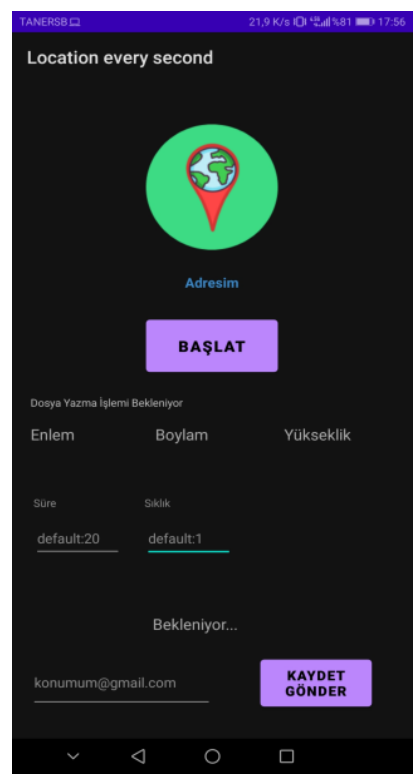

Figure 1. The interface of the 'Location Every Second' Mobile App.

The duration and sampling period value should be determined on the interface screen in Figure 1. If it is started without determination, the system will automatically define the duration as 20 minutes and the sampling period as 1 second. When the start command is given, the application saves location data as date/time, latitude, longitude and altitude both in the phone's 
documents and in itself. After the system starts, started warning of the file printing appears. After pressing the save and send button, the process stops and the e-mail function becomes active.

\subsection{Working algorithm and features of LES}

The working algorithm of this application can be summarized as follows.

1- The application is installed on the user's android phone.

2- When the application starts, it uses the GPS feature of the mobile device. For this reason, both files write permission and GPS usage permission must be given.

3- Depending on the environment used, the GNSS signal may or may not be captured. In this case, the Device cannot find the GNSS signal and will save the latitude value as 0 and the longitude value as 0 . In order for this application to record location information properly, we need to make sure that the mobile device receives GNSS signals.

4- It will record from the first second the GPS signal is captured (Mary and Bettinger, 2019). It will continue as long as the user does not end this status with the save and submit button.

5- If the device catches the GPS signal, the application will record the longitude and latitude information in the documents folder with the date of that day as the file name.

6- If desired, this file can be sent to the e-mail address entered by the mobile application user as an e-mail.

7- The next step is to clear the data. If you don't do this step, there will be a lot of data on the phone and storage space will run out. That's why we shouldn't clean our mobile tools after backing up our work.

\subsection{Application Infrastructure (Android Studio)}

While developing the application, customized integrated development environments Java coding and such as Android Studio were used. In Figure 2, the connection between the interface of the application and the features running in the background was provided, and the definitions were made. Added some functions such as a scheduler, send-Mail, high accuracy getLocation, file operation, and address data (onLocationChanged).

After the software is complete the application was first tested as a software simulation of the real hardware device, and then finally field-tested.

Real field data was collected around Izmit Bay, which was chosen as the test area, and the operation of the application was tested.

\subsection{Usage Areas of the Application}

We can list the usage areas of this application as follows:

1- Record location data in exploratory studies,

2- In search and rescue works,

3- For tracking and guiding the route followed by school buses, ambulances and cargo vehicles,

4- Data collection in mapping studies

5- For health and sports activities, can be used most effectively.

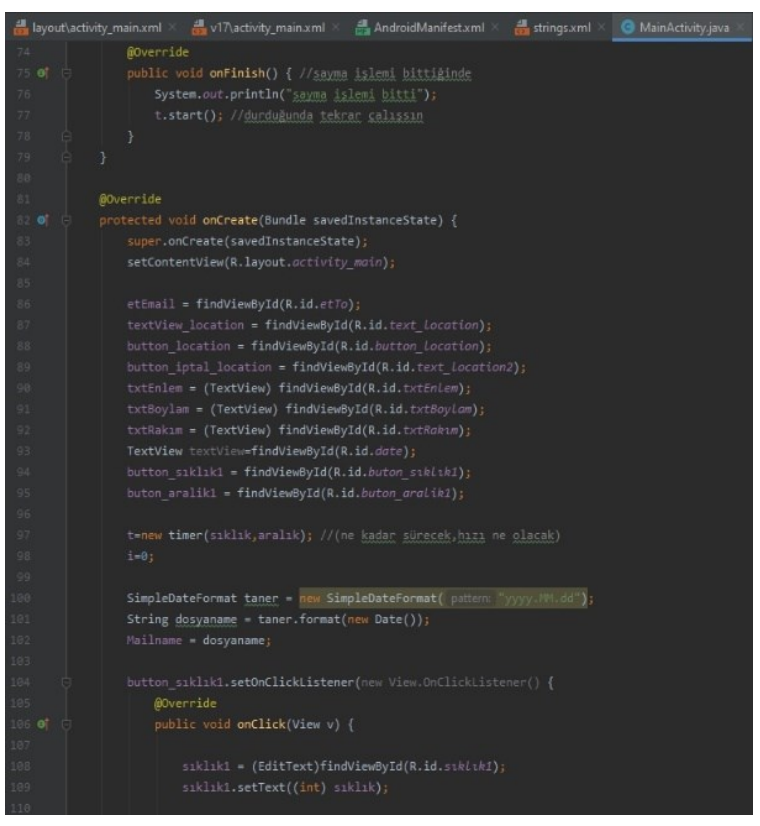

Figure 2. Coding example of the 'Location Every Second' App.

\subsection{Field Test Results}

The developed application was first tested using emulator devices, which are a software simulation of the real hardware device, and then field testing was performed. The actual field test was carried out with a car trip around the Gulf of Izmit. During the 28-minute journey, 41744.8 meters were covered. The recorded data was automatically sent to the e-mail address and the data was visualized using ARCGIS (Figure 3.).

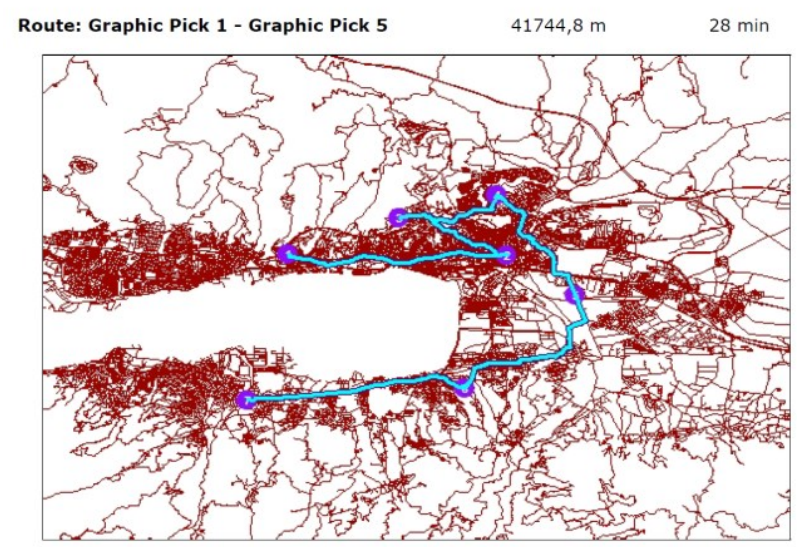

Figure 3. The route in which the test measures are performed.

\section{RESULTS}

With this application, single and dual-frequency smartphones can be used for real-time location measurements in a region selected as the study area, and their accuracy can be compared. With this application, higher accuracy location data can also be obtained using smartphones with dual-frequency receivers.

The fact that the data recording time and the recording frequency can be adjusted provides convenience for obtaining the required special size data. Fast transmission of the recorded data via internet infrastructure and e-mail allows users to make near-real-time analyses and evaluations. Additionally, the 
simple and easy interface of this application will provide ease of use and significant advantages for users.

Some future innovations for the application will be as follows:

- Adding a map base to the application and allowing instant location data to be seen on the map.

- Diversification of the data format so that we can open the recorded data easily in GIS environments.

- To be able to share data in ways other than e-mail.

\section{REFERENCES}

Amit, K. And Vineet, K., 2011. Location Based Services Using Android Mobile Operating System, Ijaft Journal, 1 (1).

Bettinger, P., Merry, K., Bayat, M. and Toma`stik, J. 2019. GNSS use in forestry - a multi-national survey from Iran, Slovakia and southern USA. Comput. Electron. Agric. 158, 369-383. doi: 10.1016/j.compag.2019.02.015.

Brach, M., Stere'nczak, K., Bolibok, L., Kwa'sny, Ł., Krok, G. and Laszkowski, M. 2019. Impacts of forest spatial structure on variation of the multipath phenomenon of navigation satellite signals. Folia For. Pol. 61, 3-21. doi: 10.2478/ffp-2019-0001.

Heitkötter, H., Hanschke, S., and Majchrzak T.A., 2013. Evaluating Cross-Platform Development Approaches For Mobile Applications. In: Springer Verlag, 2013, Pp. 120-138. Isbn: 9783642366079.

Holopainen, M., Vastaranta, M. and Hyyppa, J., 2014. Outlook for the next generation's precision forestry in Finland. Forests 5, 1682-1694. doi: 10.3390/f5071682.

Invonto, 2021. https://www.invonto.com/insights/mobile-appdevelopment-process/ (Access Date:05.07.2021)

Jobe, W., 2013. Native Apps Vs. Mobile Web Apps. In: International Journal Of Interactive Mobile Technologies (Ijim) 7.4 (2013), Pp. 27-32. Issn: 1865-7923.

Keefe, R.F., Wempe, A.M., Becker, R.M., Zimbelman, E.G., Nagler, E.S., Gilbert, S.L., 2019. Positioning methods and the use of location and activity data in forests. Forests 10. doi: 10.3390/f10050458.

Merry, K. and Bettinger, P. 2019. Smartphone GPS accuracy study in an urban environment. PLoS One 14, e0219890. doi: 10.1371 /journal. pone. 0219890 .

McGaughey, R.J., Ahmed, K., Andersen, H.E. and Reutebuch, S.E. 2017. Effect of occupation time on the horizontal accuracy of amapping-grade GNSS receiver under dense forest canopy. Photogramm. Eng. Remote Sensing 83, 861-868. doi: 10.14358/PERS.83.12.861.

Ohrt, J., Turau, V., 2012. Cross-Platform Development Tools For Smartphone Applications, Computer, 45(9), 72-79.

Paziewskia, J., Fortunato, M., Mazzoni, A., Odolinskic, R., 2021. An analysis of multi-GNSS observations tracked by recent Android smartphones and smartphone-only relative positioning results, Measurement, https://doi.org/10.1016/j.measurement.2021.109162

Robustelli, U., Baiocchi, V., Pugliano, G., 2019. Assessment of dual frequency GNSS observations from a Xiaomi Mi8 Android smartphone and positioning performance analysis. Electronics $2019,8,91$.

Sanglyne, L. J., 2015. Android Application to locate and track mobile phones (AALTM)- An implementation of location-based services, IJRET: International Journal of Research in Engineering and Technology, Volume: 04 Issue: 05, May-2015

Statista A. https://www.statista.com/statistics/263437/globalsmartphone-sales-to-end-users-since-2007/

(Access Date:05.07.2021)

Statista B., https://www.statista.com/statistics/330695/numberof-smartphone-users-worldwide/

(Access Date:05.07.2021)

Taylor, S.E., McDonald, T.P., Veal, M.W., Corley, F.W. and Grift, T.E. 2002. Precision forestry: operational tactics for today and tomorrow. In 25thAnnual Meeting of the Council of Forest Engineers. Auburn University, Auburn, Alabama Vol. 6.

Van Diggelen, F., 2009. A-GPS: Assisted GPS, GNSS, and SBAS. Artech House, Boston, pp. 350.

Warnant, R., Van De Vyvere, L., Warnant, Q., 2018. Positioning with Single and Dual Frequency Smartphones Running Android 7 or Later. In Proceedings of the 31st International Technical Meeting of the Satellite Division of The Institute of Navigation (ION GNSS+ 2018), Miami, FL, USA, 24-28 September 2018; pp. 284-303.

Wasserman, I., 2010. Software Engineering Issues For Mobile Application Development, Foser '10 Proceedings Of The Fse/Sdp Workshop On Future Of Software Engineering Research, 7-8 November 2010 New Mexico, Usa, Acm New York, Isbn: 978-1-4503-0427-6, 397- 4004

Yamakami, T., 2005. Mobile Application Platform Strategies: Business Model Engineering For The Data Intensive Mobile Age Mobile Business, 2005. Icmb 2005. International Conference On (Pp 333 - 339), (11-13 July 2005).

Zandbergen, P. A. and Barbeau, S. J. 2011. Positional accuracy of assisted GPS data from high-sensitivity GPS-enabled mobile phones. J. Navig. 64, 381-399. doi: $10.1017 / \mathrm{S} 0373463311000051$.

Zhang, K., Jiao, F., Li J., 2018. The Assessment of GNSS Measurements from Android Smartphones, in: J. Sun, C. Yang, S. Guo (Eds.), China Satellite Navigation Conference (CSNC) 2018 Proceedings, Springer Singapore, Singapore, 2018: pp. 147-157. https://doi.org/10.1007/978-981-13-0029-5_14. 